\title{
Delayed Burr Hole Surgery in Patients with Acute Subdural Hematoma : Clinical Analysis
}

\author{
Yoon Heuck Choi, M.D., Seong Rok Han, M.D., Chang Hyun Lee, M.D., Chan Young Choi, M.D., Moon Jun Sohn, M.D., \\ Chae Heuck Lee, M.D. \\ Department of Neurosurgery, Ilsan Paik Hospital, Inje University College of Medicine, Goyang, Korea
}

Objective : To evaluate the effectiveness and efficacy of delayed burr hole surgery in relation to the reduction of postoperative subdural hematoma (SDH) volume in patients with acute SDH.

Methods : We retrospectively analyzed patients with acute SDH who received delayed burr hole surgery at our institute. Age, sex, Glasgow coma scale, maximal SDH thickness, volume of SDH, midline shifts, hounsfield unit (HU), and medical history of anticoagulant agent usage were recorded. Outcome measures were delayed operation day, reduction of SDH volume after operation, and the Glasgow outcome scale (GOS) score at discharge. The patients were divided two groups according to the postoperative reduction of volume of $\mathrm{SDH}(\geq 50 \%$, group $\mathrm{A} ;<50 \%$, group B). We also analyzed variables and differences between two groups.

Results : Eighteen patients were available for this analysis. The mean delayed of surgery was $13.9 \pm 7.5$ days. Maximal thickness of $\mathrm{SDH}$ was changed from $10.0 \pm 3.5 \mathrm{~mm}$ to $12.2 \pm 3.7 \mathrm{~mm}$. Volume of SDH was changed from $38.7 \pm 28.0 \mathrm{~mL}$ to $42.6 \pm 29.6 \mathrm{~mL}$. Midline shifts were changed from $5.8 \pm 3.3 \mathrm{~mm}$ to $6.6 \pm 3.3 \mathrm{~mm}$. HU were changed from $66.4 \pm 11.2$ to $53.2 \pm 20.6$. Post-operative reduction of SDH volume was $52.1 \pm 21.1 \%$. Eleven patients (61\%) had a discharge GOS score of 1 (good recovery). Ten patients (56\%) were enrolled in group A. Midline shifting was greater in group A than in group B (7.4 \pm 3.3 vs. $3.0 \pm 2.4 \mathrm{~mm} ; p<0.02)$. The delay of surgery was shorter for group $A$ than group $B(9.2 \pm 2.3$ vs. $19.8 \pm 7.7$ days; $p<0.0008)$.

Conclusion : Among well selected patients, delayed burr hole surgery in patients with acute SDH may be effective for reduction of $\mathrm{SDH}$ volume. Further studies will be necessary to establish the effectiveness and safety of delayed burr hole surgery in patients with acute SDH.

Key Words : Subdural hematoma · Surgery.

\section{INTRODUCTION}

Traumatic subdural hematoma (SDH) is a common pathological entity in neurosurgery. Between 12\% and 29\% of patients with severe traumatic brain injury has acute $\mathrm{SDH}^{1)}$. The natural course of acute SDH differs among patients. Many patients with small acute SDH and mild neurologic deficit are managed conservatively. However, progression or conversion from acute to chronic SDH is a common cause of clinical deterioration in patients that necessitates surgical treatment ${ }^{8)}$.

- Received : April 18, 2017 • Revised : July 5, 2017 •Accepted : August 12, 2017

- Address for reprints : Seong Rok Han, M.D.

Department of Neurosurgery, Ilsan Paik Hospital, Inje University College of Medicine, 170 Juhwa-ro, Ilsanseo-gu, Goyang 10380, Korea Tel : +82-31-910-7730, Fax : +82-31-915-0885, E-mail : hsrkmj@paik.ac.kr

This is an Open Access article distributed under the terms of the Creative Commons Attribution Non-Commercial License (http://creativecommons.org/licenses/by-nc/4.0) which permits unrestricted non-commercial use, distribution, and reproduction in any medium, provided the original work is properly cited. 
With the aging of the population, neurosurgeons are faced with a growing number of elderly with acute SDH who are treated with anticoagulant medications. Open craniotomy for immediate hematoma removal is difficult in these patients). Neurosurgeons should delay surgery in such patients because of the risk of bleeding. Until now, the management of these patients has been unclear.

We retrospectively analyzed patients with acute SDH who underwent delayed burr hole surgery at our institute. The purpose of this study was to evaluate the effectiveness and efficacy of delayed burr hole surgery in relation to the reduction of postoperative $\mathrm{SDH}$ volume.

\section{MATERIALS AND METHODS}

We retrospectively reviewed and analyzed patients that underwent delayed burr hole operation initially diagnosed as acute SDH at our institute between 2010 and 2012. The scope of the study was traumatic SDH, not spontaneous SDH. All included patients were initially treated conservatively for several reasons including small SDH, old age, or anticoagulant usage. During follow-up, SDH increase or decrease in conscious patients treated by delayed burr hole surgery were analyzed.

Age, sex, Glasgow coma scale (GCS), SDH location, and medical history of anticoagulant agent usage were collected. Laboratory data were included for coagulation parameters. These included prothrombin time, international normalized ratio (INR), platelet function assay and platelet count. Maximum SDH thickness, midline shifts, volume of the SDH (length $\times$ width $\times$ depth/2) and hounsfield unit $(\mathrm{HU})$ were evaluated by initial and pre-operative brain computed tomography (CT) scanning. Outcomes were length of delayed operation in days, reduction of $\mathrm{SDH}$ volume after operation and the Glasgow outcome scale (GOS) score at discharge. Reduction of SDH volume (\%) was calculated as ([volume at preoperative SDH-SDH volume after operation]/volume at preoperative SDH $\times 100)$. Reduction of SDH volume was rated excellent ( $\geq 75 \%)$, good $(\geq 50 \%)$, fair $(\geq 25 \%)$, and poor $(<25 \%)$. The patients were divided two groups according to reduction of volume of SDH also. Reduction of SDH volume of $\geq 50 \%$ or more was defined group A. The other patients were defined as group B. Formal comparisons between two groups were made using independent t-tests. A $p$-value $<0.05$ was regarded as statistically significant. All statistical analysis was conducted using Medcalc ${ }^{\circledR}$ (ver. 9.0; Medcalc software, Broekstraat, Belgium).

\section{RESULTS}

A total of $378 \mathrm{ASDH}$ patients were admitted at our institute during the follow-up period. Among them, 18 patients were available for this analysis. In most cases, the reason for delayed burr hole operation was a progression of the SDH size with accompanying symptoms, old age and prolongation of coagulation parameters. The 18 patients comprised five women and 13 men. Mean age was $67.5 \pm 14.9$ years (range, $53-83$ ). Five patients $(28 \%)$ were taking prescribed anticoagulant medications, which included aspirin, clopidogrel and warfarin. Among them, three patients showed prolongation of coagulation parameters. In 11 patients (61\%), SDH was located at right side convexity and at the left side in seven patients (39\%). Mean GCS score was 11.1 \pm 2.0 (range, 8-14). Patients' characteristics are described in Table 1. Serial brain CT scans revealed a change in the high density SDH to low density and an increase in volume with a midline shift in the chronic SDH

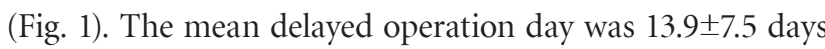
(range, 7-28 days). Pre-operatively, the mean GCS worsened to $10.6 \pm 2.5$ (range, $6-14$ ). Eight patients (44\%) patients displayed worsened GCS compared to at admission. Maximal thickness of SDH changed from $10.0 \pm 3.5 \mathrm{~mm}$ to $12.2 \pm 3.7$ $\mathrm{mm}$. Volume of SDH changed from $38.7 \pm 28.0 \mathrm{~mL}$ to $42.6 \pm$ $29.6 \mathrm{~mL}$. Midline shifts changed from $5.8 \pm 3.3 \mathrm{~mm}$ to $6.6 \pm 3.3$ $\mathrm{mm}$ (Fig. 2). HU changed from $66.4 \pm 11.2$ to $53.2 \pm 20.6$.

Post-operatively, the reduction of volume of SDH was $52.1 \pm$ $21.1 \%$. Three patients (17\%) displayed excellent results ( $\geq 75 \%$ ), seven patients (39\%) good results ( $\geq 50 \%)$, six patients (33\%) fair results ( $\geq 25 \%)$, and two patients $(11 \%)$ poor results $(<25 \%)$. Eleven patients $(61 \%)$ had a GOS score of 1 (good recovery), five patients (28\%) had a GOS score of 2 (moderate disability), and two patients (11\%) had a GOS score of 3 (severe disability) at discharge.

Ten patients (56\%) were enrolled in group A ( $\geq 50 \%$, reduction of SDH volume) and eight patients (44\%) were enrolled group B. Mean reduction of SDH volume (\%) of group A was 67.3 ( \pm 12.2$)$. Initial and pre-operative maximal thickness of 
Delayed Burr Hole Surgery of Subdural Hematoma | Choi YH, et al.

Table 1. Summary of characteristics of the patients

\begin{tabular}{|c|c|c|c|c|c|c|c|c|c|c|c|c|}
\hline \multirow[b]{2}{*}{ No. } & \multirow[b]{2}{*}{ Sex } & \multirow[b]{2}{*}{ Age } & \multirow[b]{2}{*}{$\begin{array}{c}\text { SDH } \\
\text { location }\end{array}$} & \multirow[b]{2}{*}{$\begin{array}{c}\text { Anticoagulant } \\
\text { usage }\end{array}$} & \multicolumn{5}{|c|}{ Initial } & \multirow[b]{2}{*}{$\begin{array}{c}\text { Delayed } \\
\text { operation } \\
\text { day }\end{array}$} & \multirow[b]{2}{*}{$\begin{array}{l}\text { Reduction of } \\
\text { SDH volume } \\
\text { (\%) }\end{array}$} & \multirow[b]{2}{*}{$\begin{array}{c}\text { GOS at } \\
\text { discharge }\end{array}$} \\
\hline & & & & & GCS & HU & $\begin{array}{l}\text { Maximal } \\
\text { thickness of } \\
\text { SDH (mm) }\end{array}$ & $\begin{array}{c}\text { SDH } \\
\text { volume } \\
\text { (mL) }\end{array}$ & $\begin{array}{c}\text { Midline } \\
\text { shift (mm) }\end{array}$ & & & \\
\hline 1 & F & 61 & $\mathrm{Lt}$ & - & 12 & 69 & 9.1 & 31.5 & 13.1 & 8 & 86.2 & 1 \\
\hline 2 & M & 30 & Rt & - & 8 & 62 & 5.8 & 21.4 & 6.0 & 11 & 80.3 & 3 \\
\hline 3 & F & 78 & Rt & $A+C^{*}$ & 13 & 78 & 12.3 & 19.2 & 11.9 & 13 & 80.2 & 1 \\
\hline 4 & F & 38 & Rt & $C$ & 9 & 83 & 10.2 & 19.6 & 3.5 & 8 & 70.7 & 2 \\
\hline 5 & M & 80 & $\mathrm{Lt}$ & - & 12 & 65 & 18.1 & 111.2 & 5.9 & 7 & 63.7 & 1 \\
\hline 6 & M & 80 & Rt & - & 13 & 75 & 14.1 & 23.2 & 5.7 & 13 & 68.5 & 1 \\
\hline 7 & M & 71 & Rt & - & 10 & 65 & 11.2 & 33.6 & 9.6 & 9 & 59.9 & 1 \\
\hline 8 & M & 70 & Rt & $W^{\dagger}$ & 12 & 51 & 9.1 & 28.4 & 8.0 & 8 & 61.2 & 1 \\
\hline 9 & M & 83 & $\mathrm{Lt}$ & - & 9 & 51 & 11.8 & 40.1 & 6.8 & 7 & 50.1 & 2 \\
\hline 10 & M & 72 & Rt & - & 11 & 77 & 5.7 & 113.7 & 3.3 & 8 & 52.2 & 1 \\
\hline 11 & M & 79 & $L t$ & $A^{\ddagger}$ & 13 & 75 & 11.9 & 37.1 & 3.8 & 11 & 40.3 & 2 \\
\hline 12 & M & 74 & $\mathrm{Lt}$ & A & 8 & 81 & 11.5 & 32.4 & 6.3 & 7 & 42.6 & 2 \\
\hline 13 & M & 61 & Rt & - & 10 & 79 & 9.1 & 38.7 & 7.0 & 17 & 37.4 & 1 \\
\hline 14 & M & 53 & $\mathrm{Lt}$ & - & 12 & 55 & 5.5 & 18.8 & 4.4 & 19 & 35.7 & 1 \\
\hline 15 & $M$ & 76 & Rt & - & 8 & 63 & 3.0 & 32.1 & 1.1 & 27 & 27.4 & 3 \\
\hline 16 & $F$ & 76 & Rt & - & 14 & 63 & 9.6 & 44.8 & 0.0 & 23 & 19.3 & 1 \\
\hline 17 & M & 57 & $\mathrm{Lt}$ & - & 12 & 49 & 11.0 & 20.2 & 4.2 & 26 & 13.6 & 2 \\
\hline 18 & M & 76 & Rt & - & 13 & 56 & 11.7 & 24.5 & 4.4 & 28 & 48.8 & 1 \\
\hline
\end{tabular}

*Platelet function assay, epinephrine $>300$ seconds. ${ }^{\dagger}$ Prothrombin time, international normalized ratio (INR); $3 .{ }^{*}$ Platelet function assay, adenosine diphosphate (ADP); 163 seconds. No : number, SDH : subdural hematoma, GCS : Glasgow coma scale, HU : hounsfield unit, GOS : Glasgow outcome scale, $\mathrm{F}$ : female, Lt : left convexity, $\mathrm{M}$ : male, Rt : right convexity, $\mathrm{A}$ : aspirin, C : clopidogrel, W : warfarin

$\mathrm{SDH}$ and SDH volume was greater in group A than group B. Length of delay of surgery of group A was shorter than group B (9.2 \pm 2.3 vs. $19.8 \pm 7.7$ days; $p<0.0008)$. Midline shifting was greater in group A than group B $(7.4 \pm 3.3$ vs. $3.0 \pm 2.4 \mathrm{~mm}$; $p<0.02)$. These results are summarized in Table 2 .

\section{DISCUSSION}

SDH can be divided into acute ( $<3$ days), sub-acute (3-21 days), and chronic ( $>21$ days) according to the time of the injury and the onset of clinical symptoms ${ }^{2,6)}$. Many chronic cases progress from the acute stage ${ }^{7}$. Blood in the subdural space causes an inflammatory reaction. Within a few days, fibroblasts invade the clot and form a new membrane on the inner and outer surface, followed by growth of new capillaries, enzymatic fibrinolysis and dissolution of the blood clot. Fibrin degradation products are reintegrated into new clots and inhibit hemostasis ${ }^{2,7)}$. Chronic SDH arises due to re-hemorrhage to the hematoma cavity with the osmotic gradient, generation of plasma effusion from the torn arachnoid membrane and the reabsorption of fluid $^{2,7)}$.

Three to $26 \%$ of conservatively managed acute SDH patients developed the chronic form, which requires evacuation of the hematoma. These patients may require surgery as early as 1120 days after the initial injury or as late as 3-7 months ${ }^{7-9)}$.

Rust et al. ${ }^{11)}$ reported the risk of developing chronic SDH was at least 42.5 times higher in patients who were taking warfarin and also increased for patients on aspirin, although this latter risk could not be quantified. Interestingly, Laviv 

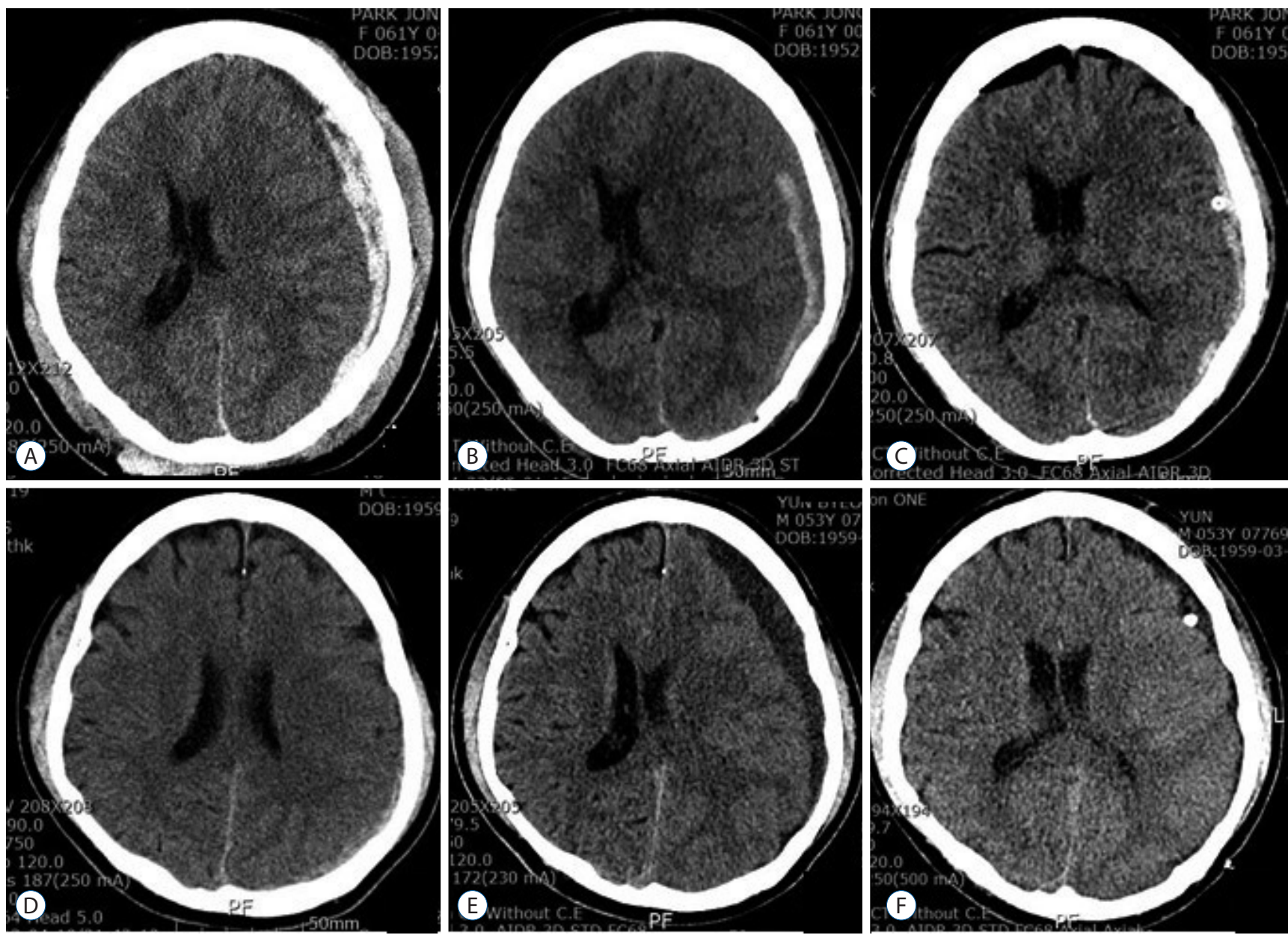

Fig. 1. Data of patients 1 and 14. Patient 1 (female/61) : the initial CT image (A) shows a thick hyper-dense ASDH at the left frontotemporoparietal convexity. The follow-up CT (B), which was performed 1 weeks after conservative treatment, reveals a hypodense SDH with an increase of midline shift and volume of SDH. Postoperative CT (C) demonstrates reduction of SDH. Patient 14 (male/53) : The initial CT image (D) reveals a thin hyperdense ASDH at the left parietoocipital convexity. The follow-up CT, which was performed 18 days, reveals a hypodense SDH with an increase of midline shift to the right hemisphere. Postoperative $\mathrm{CT}(\mathrm{F})$ shows reduction of SDH. CT : computed tomography, ASDH : acute subdural hematoma, SDH : subdural hematoma.

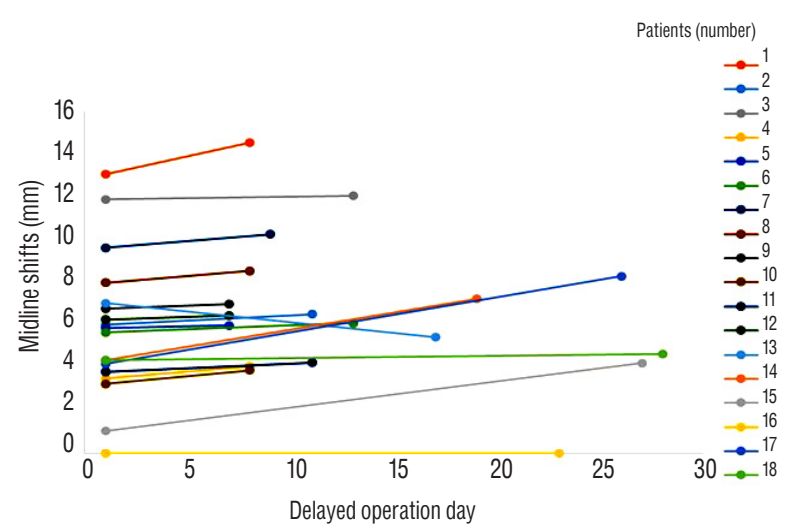

Fig. 2. Before operation, midline shifts were increased from $5.8 \pm 3.3 \mathrm{~mm}$ to $6.6 \pm 3.3 \mathrm{~mm}$. The number of patients were matched to Table 1 . and Rappaport ${ }^{7)}$ reported that the operation rate was significantly higher in patients with a medical history of ischemic heart disease or hypertension, with a 4 -fold increase in the risk for developing surgical chronic SDH in patients with ischemic heart disease and a 6-fold increased risk in patients with hypertension. Torihashi et al. ${ }^{12)}$ reported that among 343 surgical cases of chronic SDH, 61 patients experienced a recurrence of the chronic condition. Although anticoagulant therapy had no significant effect on recurrence of chronic SDH, the time interval between the injury and the first operation for patients with antiplatelet and/or anticoagulant therapy was shorter than that for patients not receiving these therapies ( 29.9 vs. 44.2 days).

There have been few reports of delayed burr hole surgery 
Table 2. Summary of characteristics of patients

\begin{tabular}{|c|c|c|c|}
\hline Characteristics & Group A $(n=10)$ & Group B $(n=8)$ & $p$-value \\
\hline Age & $66.3 \pm 18.3$ & $69.0 \pm 10.3$ & 0.14 \\
\hline Sex & & & - \\
\hline Male & $7(70)$ & $7(88)$ & \\
\hline Female & $3(30)$ & $1(12)$ & \\
\hline Delated operation day & $9.2 \pm 2.3$ & $19.8 \pm 7.7$ & 0.0008 \\
\hline \multicolumn{4}{|l|}{$\begin{array}{l}\text { Maximal thickness of } \\
\text { SDH (mm) }\end{array}$} \\
\hline Initial & $10.7 \pm 3.7$ & $9.2 \pm 3.3$ & 0.35 \\
\hline Pre-operative & $11.6 \pm 3.9$ & $12.9 \pm 2.3$ & 0.41 \\
\hline \multicolumn{4}{|l|}{ Midline shifting (mm) } \\
\hline Initial & $7.4 \pm 3.3$ & $3.0 \pm 2.4$ & 0.02 \\
\hline Pre-operative & $7.9 \pm 3.5$ & $5.1 \pm 2.5$ & 0.07 \\
\hline \multicolumn{4}{|l|}{ SDH volume (mL) } \\
\hline Initial & $44.2 \pm 36.6$ & $31.1 \pm 9.2$ & 0.34 \\
\hline Pre-operative & $48.8 \pm 38.8$ & $34.9 \pm 8.0$ & 0.34 \\
\hline $\begin{array}{l}\text { Reduction of SDH } \\
\text { volume (\%) }\end{array}$ & $67.3 \pm 12.2$ & $33.1 \pm 12.1$ & - \\
\hline
\end{tabular}

Values are presented as mean \pm standard deviation or number (\%). SDH : subdural hematoma

involving acute SDH patients. Izumihara et al. ${ }^{3)}$ reported that eight acute SDH patients required surgery in the sub-acute and chronic stage. Among them, six patients (75\%) underwent burr hole surgery and mean time of delayed surgery was 20 days (range, 16-23). Godlewski et al. ${ }^{2}$ reported on operative treatment of a series of 100 patients with SDH. Among them, 50 patients (50\%) underwent burr hole surgery after sub-acute $(n=15,30 \%)$ or chronic stage $(n=35,70 \%)$. Mathew et al. ${ }^{10)}$ reported on 15 patients who required delayed burr hole evacuation of their acute SDH. The mean time between initial injury and operation was 14 days (range, 11-20 days). Kim et al. ${ }^{5)}$ reported on delayed surgical evacuation for initially non-operative acute SDH. Sixty four patients (65\%) were treated with conservative management and 34 patients $(35 \%)$ required delayed hematoma evacuation of median of 17 days (range, 6-39 days). Twenty two patients (65\%) required surgical intervention within 3 weeks of head injury (sub-acute stage) and the remaining 12 patients (35\%) underwent burr hole surgery more than 3 weeks after injury (chronic stage). The authors reported that two prognostic factors, including the initial volume of the ASDH and the degree of midline shift on the ini- tial CT scan, were independently associated with delayed hematoma evacuation for $\mathrm{ASDH}^{5}$.

Delayed mean operation day of our series was 13.9 days (range, 7-28 days), similar to the aforementioned studies. In view of SDH reduction of after operation, patients with greater post-operative reduction of $\mathrm{SDH}$ volume (group A) experience a shorter delay of surgery ( $9.2 \pm 2.3$ days) than group B (19.8 \pm 7.7 days). Midline shifting, maximal thickness of SDH and SDH volume were greater in group A than group B. We think that more shorter delay operation day in group A than group B is influenced by more larger SDH in group A. Delayed burr hole surgery is generally effective and successful in chronic stage. Our series show that among well selected patients, delayed burr hole surgery in patients with acute SDH may be effective for reduction of SDH volume at late subacute stage and chronic stage.

\section{CONCLUSION}

We analyzed delayed burr hole surgery in patients with initially presented acute SDH. Among well selected patients, delayed burr hole surgery in such patients may be effective for reduction of SDH volume. However, more studies are needed to conclusively establish the effectiveness and safety of delayed burr hole surgery in patients with acute SDH.

\section{References}

1. Bajsarowicz P, Prakash I, Lamoureux J, Saluja RS, Feyz M, Maleki M, et al : Nonsurgical acute traumatic subdural hematoma: what is the risk? J Neurosurg 123 : 1176-1183, 2015

2. Godlewski B, Pawelczyk A, Pawelczyk T, Ceranowicz K, Wojdyn M, Radek $\mathrm{M}$ : Retrospective analysis of operative treatment of a series of 100 patients with subdural hematoma. Neurol Med Chir (Tokyo) 53 26-33, 2013

3. Izumihara A, Yamashita K, Murakami T : Acute subdural hematoma requiring surgery in the subacute or chronic stage. Neurol Med Chir (Tokyo) 53 : 323-328, 2013

4. Karibe H, Hayashi T, Hirano T, Kameyama M, Nakagawa A, Tominaga T : Surgical management of traumatic acute subdural hematoma in adults: a review. Neurol Med Chir (Tokyo) $54:$ 887-894, 2014

5. Kim BJ, Park KJ, Park DH, Lim DJ, Kwon TH, Chung YG, et al. : Risk factors of delayed surgical evacuation for initially nonoperative acute subdural hematomas following mild head injury. Acta Neurochir (Wien) 
$156: 1605-1613,2014$

6. Kuwahara S, Fukuoka M, Koan Y, Miyake H, Ono Y, Moriki A, et al. : Diffusion-weighted imaging of traumatic subdural hematoma in the subacute stage. Neurol Med Chir (Tokyo) 45 : 464-469, 2005

7. Laviv $Y$, Rappaport ZH : Risk factors for development of significant chronic subdural hematoma following conservative treatment of acute subdural hemorrhage. Br J Neurosurg 28 : 733-738, 2014

8. Lee JJ, Won Y, Yang T, Kim S, Choi CS, Yang J : Risk factors of chronic subdural hematoma progression after conservative management of cases with initially acute subdural hematoma. Korean J Neurotrauma 11 : 52-57, 2015

9. Lindvall P, Koskinen LO : Anticoagulants and antiplatelet agents and the risk of development and recurrence of chronic subdural haematomas. J Clin Neurosci 16 : 1287-1290, 2009

10. Mathew $P$, Oluoch-Olunya DL, Condon BR, Bullock R : Acute subdural haematoma in the conscious patient: outcome with initial non-operative management. Acta Neurochir (Wien) 121 : 100-108, 1993

11. Rust $T$, Kiemer $N$, Erasmus $A$ : Chronic subdural haematomas and anticoagulation or anti-thrombotic therapy. J Clin Neurosci 13 : 823-827, 2006

12. Torihashi K, Sadamasa N, Yoshida K, Narumi O, Chin M, Yamagata S Independent predictors for recurrence of chronic subdural hematoma: a review of 343 consecutive surgical cases. Neurosurgery 63 : 11251129; discussion 1129, 2008 